\author{
SUBJECT AREAS: \\ NON-SMALL-CELL LUNG \\ CANCER \\ TARGETED THERAPIES \\ CANCER GENETICS \\ CHEMOTHERAPY
}

Received

20 August 2014

Accepted

3 November 2014

Published

24 November 2014

Correspondence and requests for materials should be addressed to Y.S. (Sun_yihua76@ hotmail.com) or H.C. (hqchen1@yahoo. com)

* These authors contributed equally to this work.

\section{The prognostic and predictive value of solid subtype in invasive lung adenocarcinoma}

\author{
Yang Zhang ${ }^{1,2 *}$, Jing Li ${ }^{1,2 *}$, Rui Wang ${ }^{1,2 *}$, Yuan Li ${ }^{2,3}$, Yunjian Pan ${ }^{1,2}$, Deng Cai ${ }^{1,2}$, Haichuan $\mathrm{Hu}^{1,2}$, \\ Hang Li ${ }^{1,2}$, Ting Ye ${ }^{1,2}$, Xiaoyang Luo ${ }^{1,2}$, Yiliang Zhang ${ }^{1,2}$, Bin Li ${ }^{1,2}$, Lei Shen ${ }^{2,3}$, Yihua Sun ${ }^{1,2}$ \\ \& Haiquan Chen ${ }^{1,2}$
}

\begin{abstract}
'Department of Thoracic Surgery, Fudan University Shanghai Cancer Center, Shanghai, 200032, China, ${ }^{2}$ Department of Oncology, Shanghai Medical College, Fudan University, Shanghai, 200032, China, ${ }^{3}$ Department of Pathology, Fudan University Shanghai Cancer Center, Shanghai, 200032, China.
\end{abstract}

A total of 1039 stage I-III invasive lung adenocarcinoma including 186 solid subtype patients who have undergone radical resection were assessed for clincopathlogic characteristics, status of common driver mutations, pattern of recurrence, recurrence-free survival (RFS), overall survival (OS), post-recurrence survival (PRS) and predictive value for adjuvant chemotherapy and EGFR tyrosine kinase inhibitors (TKIs). Solid predominant adenocarcinomas were more likely to have initial distant recurrences than non-solid subtype invasive adenocarcinomas $(P=0.018)$. In univariate analysis, solid predominant adenocarcinoma patients had significantly worse RFS $(P<0.001)$, OS $(P<0.001)$ and PRS $(P=0.010)$. Multivariate analysis adjusting for clinicopathologic variables and mutational status showed that solid subtype was an independent poor prognostic factor (odds ratio $=1.876,95 \%$ confidence interval: $1.291-3.158 ; P=0.003$ ) and an independent negative predictor for stage II-III patients undergoing adjuvant chemotherapy (odds ratio $=2.020,95 \%$ confidence interval: $1.291-3.158 ; P=0.002$ ). In $E G F R$-mutated solid predominant lung adenocarcinoma patients who experienced disease recurrence, the response rate to EGFR TKIs was only $37.5 \%$. In radically resected invasive lung adenocarcinoma, solid subtype was an independent poor prognostic factor and negative predictor for adjuvant chemotherapy.

ung adenocarcinoma is a markedly heterogeneous entity characterized by various histologic subtypes as well as clinically relevant oncogenic driver mutations. Since the release of the new lung adenocarcinoma clas- sification proposed by the International Association for the Study of Lung Cancer/American Thoracic Society/European Respiratory Society (IASLC/ATS/ERS) ${ }^{1}$, many studies have investigated the possible correlations among predominant adenocarcinoma subtype, driver mutations and patient prognosis ${ }^{2-9}$.

Solid predominant lung adenocarcinoma were reported to be associated with aggressive clinicopathologic features, including larger tumor size at presentation, lymph node metastasis as well as lymphovascular and pleural invasion $^{6,10-12}$. It is reported that solid pattern is a poor prognostic factor in lung adenocarcinoma ${ }^{2,4,6,8,12,13}$. However, the predictive value of solid pattern for chemotherapy/radiotherapy remains poorly defined ${ }^{2,3,7}$. A recent study also suggested that solid subtype was a negative predictor of response to EGFR tyrosine kinase inhibitor (TKI) treatment ${ }^{14}$.

In this study, we performed a comprehensive analysis of solid predominant lung adenocarcinoma regarding clincopathologic characteristics, molecular status, and most importantly, the prognostic and predictive value.

\section{Results}

A total of 186 solid predominant lung adenocarcinoma patients were included in this study, along with 522 acinar predominant, 143 papillary predominant, 113 lepidic predominant, 20 micropapillary predominant, 2 enteric predominant and 53 invasive mucinous adenocarcinoma. There were 481 male patients and 558 female patients. The median age was 60 years old, with a range of 22 to 84 years. The number of cases in stages I, II and III were 580 , 146 and 313, respectively (Table 1).

Solid predominant adenocarcinoma was significantly correlated with male gender $(P<0.001)$, younger age at diagnosis $(P=0.049)$ and smoking history $(P<0.001)$. More solid subtype patients underwent resections greater 


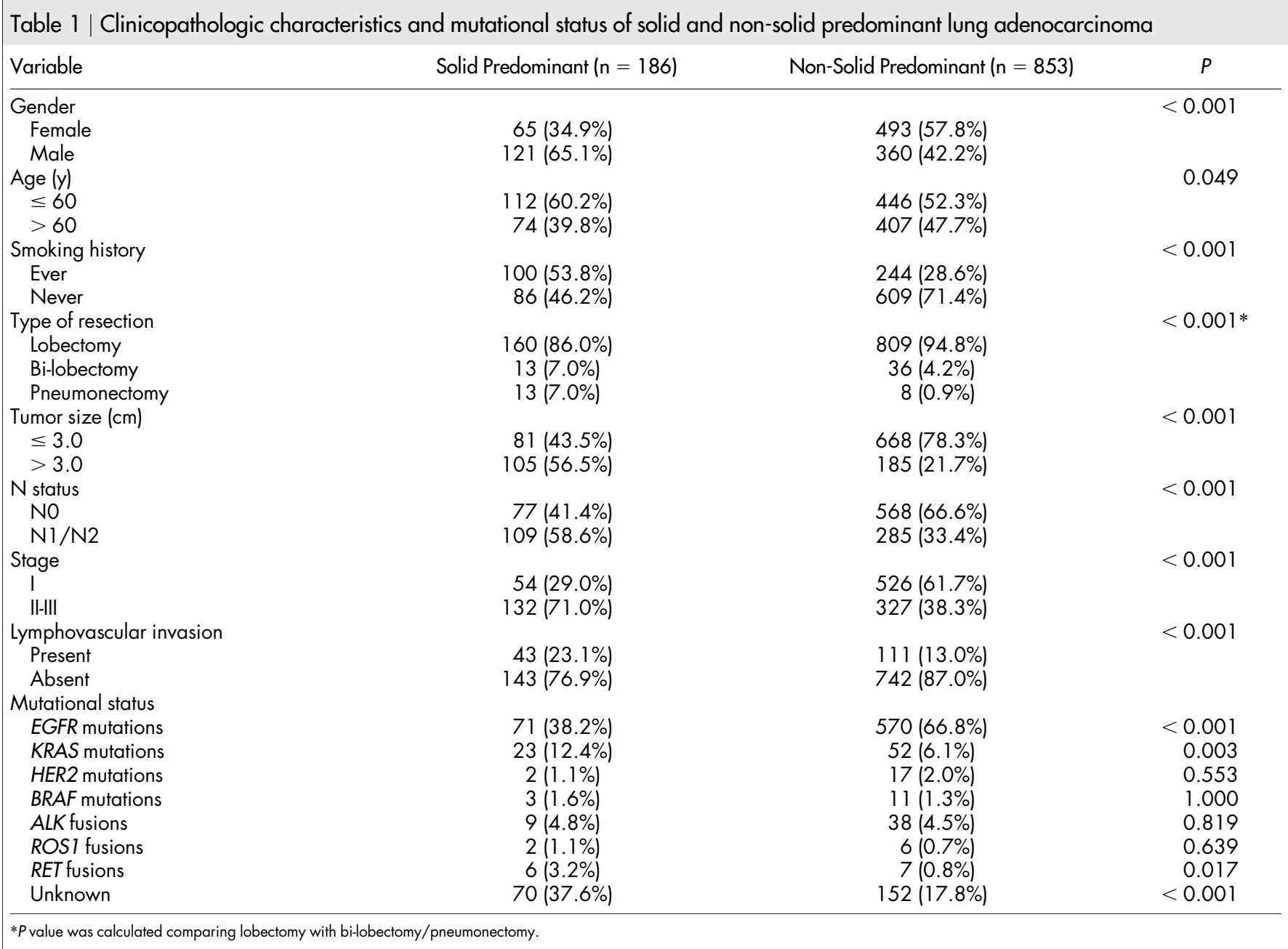

than lobectomy (bi-lobectomy or pneumonectomy). Solid subtype adenocarcinoma was significantly associated with aggressive tumor characteristics including larger tumor size $(P<0.001)$, higher prevalence of lymphovascular invasion $(P<0.001)$ and lymph node metastasis $(P<0.001)$, and more advanced disease stage $(P<0.001)$ (Table 1).

Compared to other histologic subtypes, solid predominant adenocarcinoma harbored significantly lower frequency of EGFR mutations (38.2\% vs. $66.8 \%, P<0.001)$, but higher prevalence of $K R A S$ mutations $(12.4 \%$ vs. $6.1 \%, P=0.003)$ and $R E T$ fusions $(3.2 \%$ vs. $0.8 \%, P=0.017)$. Significantly higher proportion of solid predominant adenocarcinomas were "pan-negative" for these 7 common driver mutations ( $37.6 \%$ vs. $17.8 \%, P<0.001$ ) (Table 1 ).

To ensure a sufficient follow up, we included 442 patients diagnosed between October 2007 and March 2011 for survival analysis. The patient cohort were comprised of 94 solid predominant, 211 acinar predominant, 75 papillary predominant, 36 lepidic predominant, 8 micropapillary predominant, 2 enteric predominant, and 16 invasive mucinous adenocarcinoma.

In univariate analysis, solid predominant adenocarcinoma patients had significantly worse RFS $(P<0.001)$ and OS $(P<$ 0.001 ) than non-solid subtype (Figure $1 \mathrm{~A}$ and $1 \mathrm{~B}$ ). A total of 63 solid subtype and 155 non-solid subtype patients experienced disease recurrence. When comparing pattern of recurrence, we found that distant recurrence was more likely to occur in solid predominant adenocarcinoma ( $82.5 \%$ vs. $66.5 \%, P=0.018$ ).

When the survival analysis was limited to stage II-III patients (Figure 1E and 1F), the survival difference between solid and nonsolid subtype was still significant regarding RFS $(P=0.011)$ and OS
$(P=0.001)$. When stage II and stage III patients were analyzed separately, solid predominant lung adenocarcinoma patients had significantly worse OS in both category $(P=0.019$ for stage II; $P$ $=0.031$ for stage III). However, statistical significance was not achieved when comparing RFS $(P=0.206)$ or OS $(P=0.498)$ in stage I patients (Figure 1C and 1D).

In multivariate survival analysis incorporating gender, age, smoking history, lymphovascular invasion, pathologic stage (stage II/III vs. stage I), histologic subtype and mutational status (Table 2), solid subtype was an independent predictor of poor OS (OR $=1.876,95 \%$ CI: $1.291-3.158 ; P=0.003$ ).

As the application of postoperative adjuvant chemotherapy in stage I NSCLC remains controversial, we therefore investigated the predictive value of solid subtype for stage II-III adenocarcinoma patients who received adjuvant chemotherapy. In univariate analysis (Figure $1 \mathrm{G}$ and $1 \mathrm{H}$ ), solid subtype was significantly associated with worse RFS $(P=0.015)$ and OS $(P=0.001)$.

In multivariate survival analysis adjusting for gender, age, smoking history, lymphovascular invasion, pathologic stage (stage III vs. stage II), histologic subtype and mutational status (Table 2), solid subtype also proved to be an independent predictor of poor OS (OR $=2.020$, 95\% CI: $1.291-3.158 ; P=0.002)$.

In univariate analysis (Figure 2), solid subtype patients had significantly worse PRS than non-solid predominant adenocarcinoma patients $(P=0.010)$. However, pathologic stage and EGFR mutational status, but not solid histology, were independent predictors of PRS as demonstrated in multivariate analysis (Table 2).

Of the 442 invasive lung adenocarcinoma patients diagnosed between October 2007 and March 2011, eight EGFR-mutated solid 
A

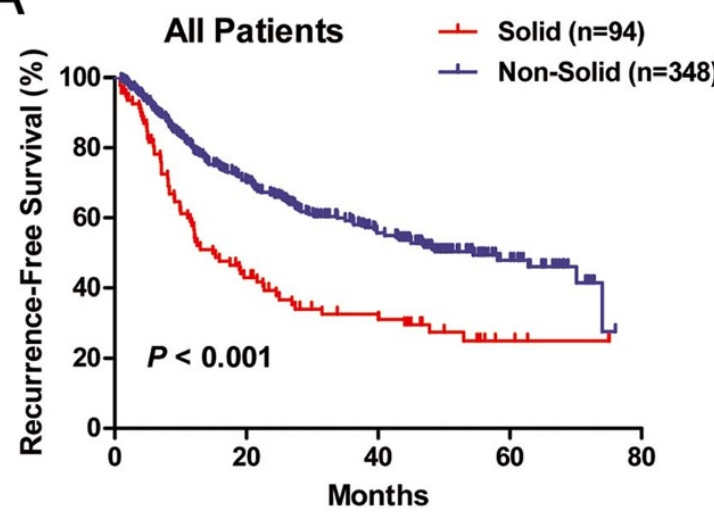

C

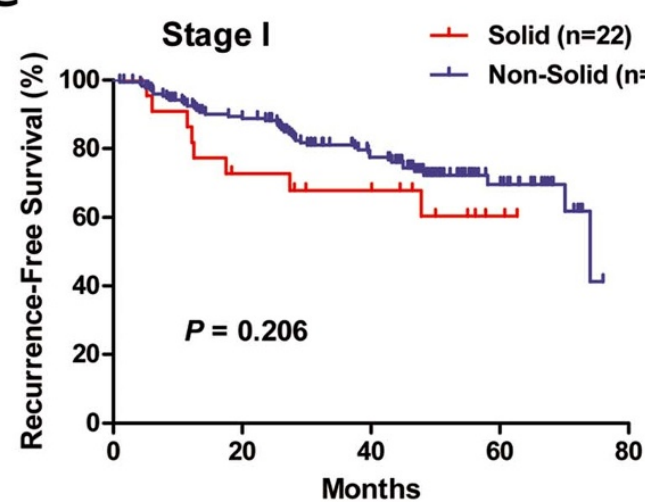

E

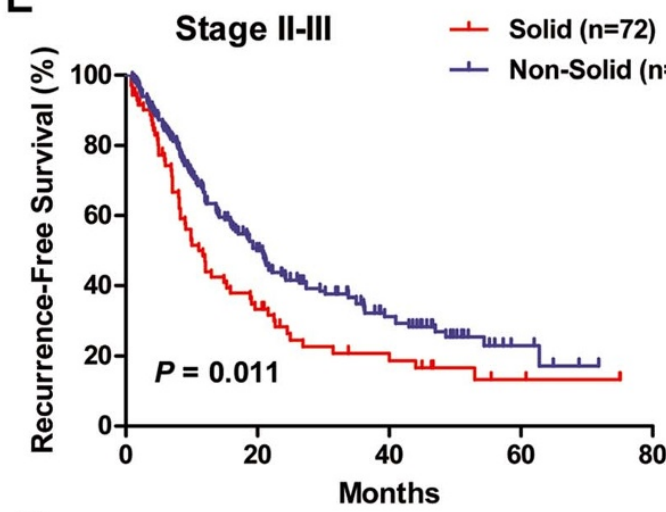

G

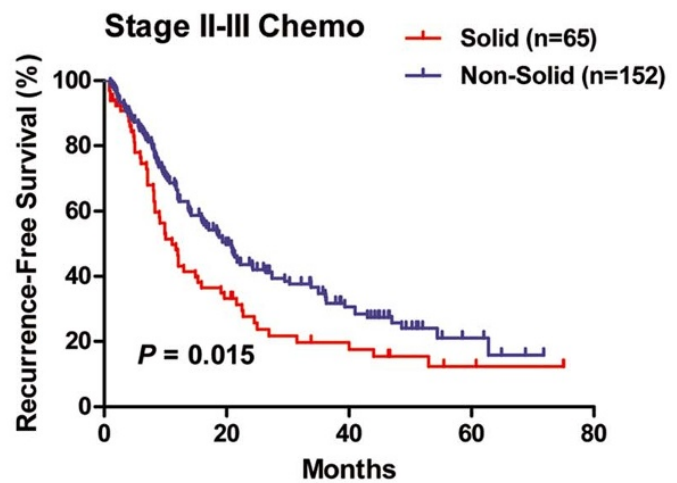

B

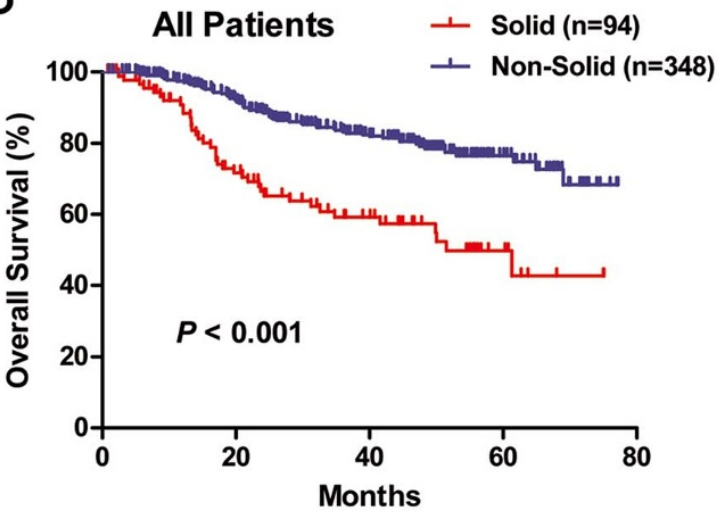

D

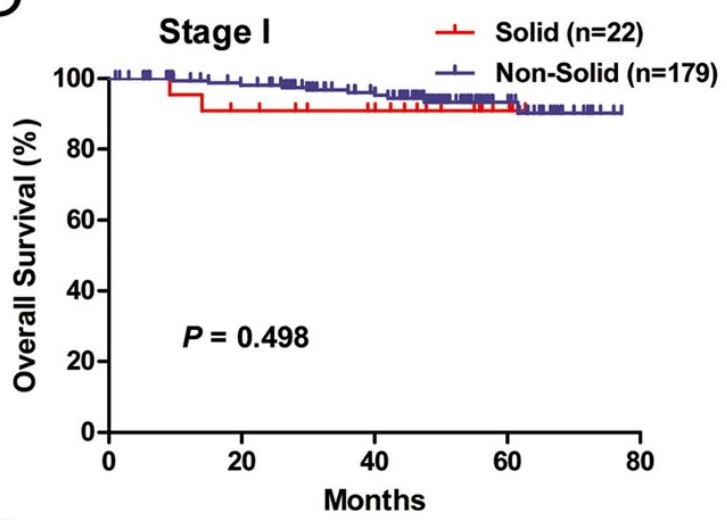

F

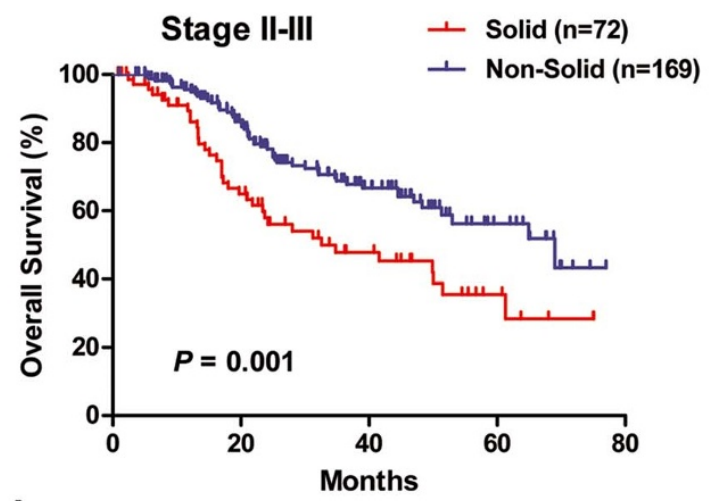

$\mathrm{H}$

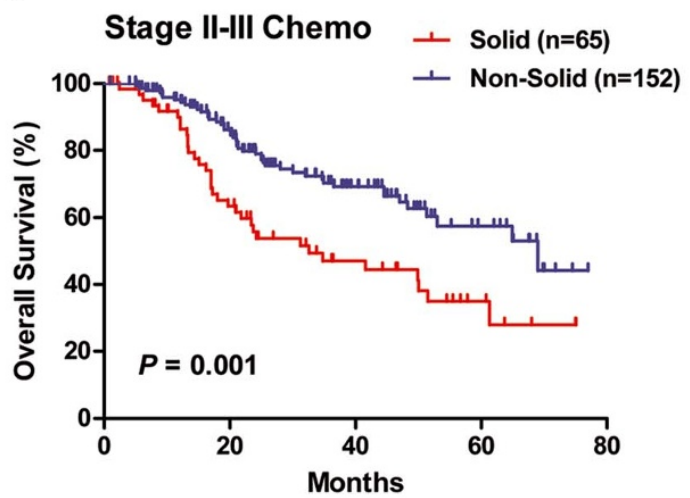

Figure $1 \mid$ Recurrence-free survival (RFS) and overall survival (OS) of all patients (A and B), stage I patients (C and D), stage II-III patients (E and F) and stage II-III patients undergoing postoperative adjuvant chemotherapy $(\mathrm{G}$ and $\mathrm{H})$ between solid and non-solid predominant lung adenocarcinoma. 
Table 2 | Independent predictors of overall survival, post-recurrence survival of all patients and survival outcome of stage II-III patients undergoing adjuvant chemotherapy in multivariate analysis

Overall Survival: All patients

\begin{tabular}{|c|c|c|c|c|}
\hline Variable & Category & OR & $95 \% \mathrm{Cl}$ & $P$ \\
\hline \multicolumn{5}{|c|}{ Overall Survival: Stage II-III patients undergoing adjuvant chemotherapy } \\
\hline \multicolumn{5}{|c|}{ Post-Recurrence Survival: All patients } \\
\hline Variable & Category & OR & $95 \% \mathrm{Cl}$ & $P$ \\
\hline
\end{tabular}

predominant lung adenocarcinoma patients received EGFR TKIs (gefitinib or erlotinib) after disease recurrence. Detailed information was listed in Table 3. There were three partial response (PR), two stable disease (SD) and three progressive disease (PD). The objective response rate was $37.5 \%$.

The NF1 mRNA expression was analyzed in 571 lung adenocarcinoma samples harboring classic EGFR mutations including exon 19 deletions and L858R as well as normal lung tissues. Low expression level of NF1 was found in 33 out of $65(50.8 \%)$ solid predominant adenocarcinomas and 315 out of $506(62.3 \%)$ non-solid predominant tumors. There was no significant correlation between solid predominant tumors and NF1 expression $(P=0.074)$.

\section{Discussion}

The correlations among the histologic subtype of lung adenocarcinoma, the status of common driver mutations, patient prognosis and the predictive value for TKIs as well as cytotoxic chemotherapy treatment have recently been investigated worldwide to optimize the treatment strategy for this disease. In this study, we reviewed a large sample of completely resected invasive lung adenocarcinoma to have a comprehensive assessment of the clinicopathologic characteristics, mutational status, patterns of recurrence, RFS, OS and PRS

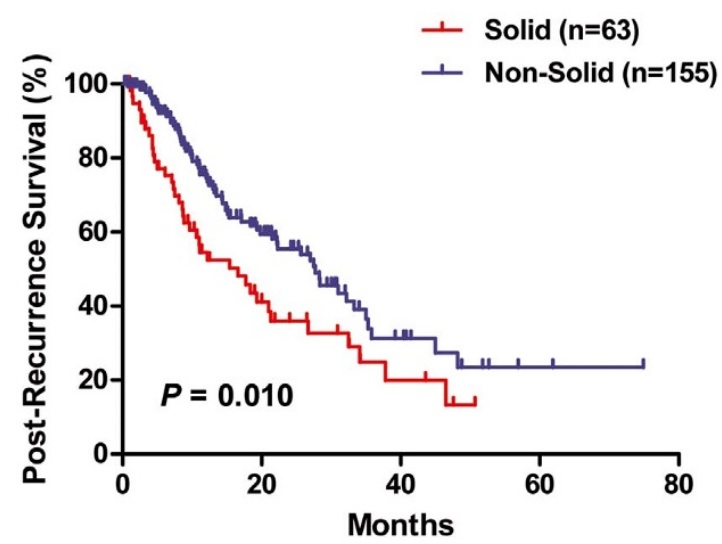

Figure 2 Post-recurrence survival (PRS) between solid and non-solid predominant lung adenocarcinoma. associated with solid predominant adenocarcinoma as well as its predictive value for postoperative adjuvant chemotherapy and EGFR TKIs after recurrence.

Consistent with previous studies ${ }^{6,10-12}$, we also found that solid predominant adenocarcinoma patients were more likely to be males and ever smokers, and were characterized by more advanced TNM stage. Molecularly, solid subtype adenocarcinomas were negatively associated with EGFR mutations, while positively correlated with KRAS mutations and RET fusions. More than one third (37.6\%) of solid predominant lung adenocarcinoma could not be defined by any of the seven common driver mutations, about twice the proportion of other invasive adenocarcinoma subtypes. More attention should be paid to this aggressive lung adenocarcinoma subtype to identify new molecular targets.

Our study showed that patients with solid predominant adenocarcinomas were more likely to have initial distant recurrences than non-solid invasive adenocarcinomas. This result is in consistence with a previous study by Hung and colleagues ${ }^{2}$, probably due to the more aggressive behavior of solid subtype.

The poor prognostic role of solid histology has been reported by several previous studies ${ }^{2,4,6,8,10,12,13}$. We further showed that solid subtype was an independent poor prognostic marker by multivariate survival analysis incorporating clinicopathologic variables as well as status of well-identified driver mutations. When the survival analysis was stratified by pathologic stage, we found that solid subtype was associated with shorter RFS and OS in stage II-III patients, but not in stage I patients. One explanation is that in this study, we excluded AIS and MIA which are usually classified as stage I and have extremely good prognosis according to previous studies ${ }^{8,15}$. The relatively small number of stage I solid predominant adenocarcinoma patients and the short follow up is another possible explanation. Finally, as postoperative adjuvant chemotherapy was routinly recommended for stage II-III paitnets who could tolerate, the survival difference between solid and non-solid subtype might be enlarged if solid predominant adencarcinomas were relatively insensitive to cytotoxic chemotherapy.

The predictive value of solid subtype for adjuvant chemotherapy/ radiotherpay remains controversial ${ }^{2,3,7}$. In a German cohort of 500 stage I-IV resected lung adenocarcinomas, the authors found that solid predominant adenocarcinoma patients had improved prognosis with adjuvant radiotherapy ${ }^{7}$. In a Mexico cohort of 313 stage 


\begin{tabular}{|c|c|c|c|c|c|c|c|}
\hline No. & Age (y) & Sex & Smoking History & Stage & Mutation & EGFR TKIs & Response \\
\hline 1 & 59 & $\mathrm{~F}$ & Never & IIIA & E19 del & Gefitinib & PD \\
\hline 2 & 54 & $\mathrm{~F}$ & Never & $\mathrm{IA}$ & E19 del & Gefitinib & PR \\
\hline 3 & 61 & $\mathrm{~F}$ & Never & IIIA & L858R & Gefitinib & PD \\
\hline 4 & 52 & M & Ever & IIIA & L858R & Erlotinib & SD \\
\hline 5 & 58 & $\mathrm{~F}$ & Never & IIIA & L858R & Gefitinib & PD \\
\hline 6 & 61 & $M$ & Ever & IIIA & L858R & Gefitinib & SD \\
\hline 7 & 56 & $\mathrm{~F}$ & Never & IIIA & E19 del & Gefitinib & PR \\
\hline 8 & 55 & $\mathrm{~F}$ & Never & $\mathrm{IB}$ & E19 del & Gefitinib & PR \\
\hline
\end{tabular}

Abbreviations: $F$, female; $M$, male; E19 del, exon 19 deletions; TKls, tyrosine kinase inhibitors; PR, partial response; SD, stable disease; PD, progressive disease.

IIIB-IV lung adenocarcinoma, Campos-Parra and collegues ${ }^{3}$ revealed that high-grade lung adenocarcinoma (solid, micropapillary and papillary-predomiannt) patients had better response rates, progression-free survival and overall survival than lepidic and acinarpredomiannt adenocarcinoma patients when treated with platinumbased chemotherapy. However, a recent study of an Asian cohort of 573 stage I-III lung adenocarcinoma patients reported that solid predominant adenocarcinoma was a poor predictor in patients undergoing adjuvant chemotherapy ${ }^{2}$. In this study, we performed multivariate survival analysis of stage II-III patients who received postoperative adjuvant chemotherapy, and found that solid subtype was an independent predictor of poor OS.

We further showed that solid predominant adencarcinoma was significantly correlated with poor post-recurrence survival in univariate analysis. However, multivariate analysis revealed that more advanced disease stage and wild-type EGFR mutational status, rather than solid histology, were independent predictors of poor PRS, indicating that the poor PRS of patients with solid predominant might be in part due to the relatively lower frequency of EGFR mutations in this histologic subtype and therefore less opportunity to receive targeted therapies. Yoshida and colleagues ${ }^{14}$ reported that solid predominant histology was negatively associated with response to EGFR TKIs in EGFR-mutated lung adenocarcinoma patients compared to other histologic subtypes (response rate: $61 \%$ vs. $88 \%, P=$ $0.03)$. In this study, we observed a response rate of only $37.5 \%(3 / 8)$, while a PD rate of $37.5 \%$ (3/8) in EGFR-mutated solid predominant lung adenocarcinoma patients with recurrent diesease. However, the number of patients is still too small to draw a definite conclusion.

A recent study revealed that low expression level of NF1 was correlated with primary and acquired resistance of lung adenocarcinms to EGFR TKIs ${ }^{16}$. In 2014, the Cancer Genome Atlas Research Network released the comprehensive molecular profiling of 230 lung adenocarcinomas. One of their findings was that the proximalinflammatory subtype of lung adenocarcinoma, which was charaterized by solid histopathology, was associated with loss-of-function mutations of $\mathrm{NF}^{17}$. However, we did not find a significant association between solid subtype and NF1 expression, suggesting that the expression level of NF1 might not be responsible for the relatively poor response to EGFR TKIs of solid predominant lung adenocarcinoma patients harboring EGFR mutations.

In conclusion, solid subtype was an independent poor prognostic factor and an independent negative predictor for adjuvant chemotherapy in invasive lung adenocarcinoma patients undergoing radical resection. In addition to the relatively lower frequency of $E G F R$ mutations in solid predominant lung adenocarcinoma, EGFR-mutated solid subtype patients with recurrent disease had poor response to EGFR TKIs. Future studies are warranted to identify novel therapeutic targets to benefit more patients with this aggressive disease.

\section{Methods}

This study included lung adenocarcinoma consecutively resected between October 2007 and May 2013 at the Department of Thoracic Surgery, Fudan University
Shanghai Cancer Center. Inclusion criteria for this study were: (1) invasive lung adenocarcinoma; (2) patients underwent complete resection (lobectomy or greater extent) with curative intent. Adenocarcinoma in situ (AIS) and minimally invasive adenocarcinoma (MIA) were excluded. Patients who had a history of malignancy or received neoadjuvant chemotherapy were excluded. Patients who received sublobectomy (including wedge resection and segmentectomy) or palliative surgeries were also excluded.

Pathologic sections were re-reviewed by two pathologists (Yuan Li and Lei Shen), and the predominant histologic subtype of lung adenocarcinoma was recorded according to the IASLC/ATS/ERS classification system ${ }^{1}$. Clinicopathologic data collected included age, gender, smoking history, type of surgical resection, lymphovascular invasion and pathologic tumor-node-metastasis (TNM) stage according to the $7^{\text {th }}$ edition of the lung cancer staging system ${ }^{18}$. Local recurrence was defined as disease recurrence in the ipsilateral hemithorax and mediastinum. Distant recurrence was defined as recurrence in the contralateral lung or outside the hemithorax and mediastinum. The recurrence was defined as "distant" if both local and distant recurrences were detected within 3 months. Recurrence-free survival (RFS) and overall survival (OS) was obtained based upon follow up clinic or telephone.

RNA was extracted from frozen tumor samples, and was reverse transcribed into cDNA. Direct dideoxynucleotide sequencing was performed to detect mutations in EGFR (exons 18-22), HER2 (exons 18-21), KRAS (exons 2-3) and BRAF (exons 11$15)$ using PCR-amplified products. The detection of $A L K, R O S 1$ and RET fusions was performed using a combination strategy of quantitative real-time PCR (qRT-PCR) and reverse transcriptase PCR (RT-PCR) ${ }^{19,20}$.

NF1 mRNA expression was measured by qRT-PCR using cDNA extracted from lung adenocarcinomas harboring classic EGFR mutations including exon 19 deletions and L858R as well as normal lung samples. The expression level of the housekeeping gene $\beta$-actin was measured as an internal reference. Normalized expression value of NF1 was calculated using the formula: $2^{[\text {CT (Normal) - CT ( } \beta \text {-actin)] - [CT (Tumor)- CT ( } \beta \text {-actin)] }}$. A NF1 expression value of $>1.0$ was considered as "high expression"; otherwise, it was defined as "low expression".

The correlations between solid predominant subtype and clinicopathologic features as well as mutational status and NF1 expression levels were assessed using Pearson's chi-squared test or Fisher's exact test. Comparisons of RFS, OS or postrecurrence survival (PRS) were conducted using the Kaplan-Meier method with logrank test in univariate analysis. Multivariate survival analysis was performed using the Cox proportional hazards regression (forward likelihood ratio model) to estimate the odds ratio (OR) and 95\% confidence interval (CI). The statistical analysis was conducted in SPSS 16.0 (SPSS Inc, Chicago, Ill). All tests were two tailed, and $P<0.05$ was considered as statistically significant.

Ethics Statement. This study was conducted in line with the Helsinki Declaration, and was approved by the Institutional Review Board of the Fudan University Shanghai Cancer Center. Written informed consent was obtained from each patient to allow their biological samples to be genetically analyzed. The experimental protocol of this study was performed strictly in accordance to the guidelines.

1. Travis, W. D. et al. International association for the study of lung cancer/american thoracic society/european respiratory society international multidisciplinary classification of lung adenocarcinoma. J Thorac Oncol 6, 244-285 (2011).

2. Hung, J. J. et al. Predictive Value of the International Association for the Study of Lung Cancer/American Thoracic Society/European Respiratory Society Classification of Lung Adenocarcinoma in Tumor Recurrence and Patient Survival. J Clin Oncol (2014).

3. Campos-Parra, A. D. et al. Relevance of the novel IASLC/ATS/ERS classification of lung adenocarcinoma in advanced disease. Eur Respir J 5, 1439-1447 (2014).

4. Yoshizawa, A. et al. Validation of the IASLC/ATS/ERS lung adenocarcinoma classification for prognosis and association with EGFR and KRAS gene mutations: analysis of 440 Japanese patients. J Thorac Oncol 8, 52-61 (2013).

5. Russell, P. A. et al. Correlation of mutation status and survival with predominant histologic subtype according to the new IASLC/ATS/ERS lung adenocarcinoma classification in stage III (N2) patients. J Thorac Oncol 8, 461-468 (2013). 
6. Hung, J. J. et al. Prognostic value of the new International Association for the Study of Lung Cancer/American Thoracic Society/European Respiratory Society lung adenocarcinoma classification on death and recurrence in completely resected stage I lung adenocarcinoma. Ann Surg 258, 1079-1086 (2013).

7. Warth, A. et al. The novel histologic International Association for the Study of Lung Cancer/American Thoracic Society/European Respiratory Society classification system of lung adenocarcinoma is a stage-independent predictor of survival. J Clin Oncol 30, 1438-1446 (2012).

8. Yoshizawa, A. et al. Impact of proposed IASLC/ATS/ERS classification of lung adenocarcinoma: prognostic subgroups and implications for further revision of staging based on analysis of 514 stage I cases. Mod Pathol 24, 653-664 (2011).

9. Zhang, Y. et al. Frequency of driver mutations in lung adenocarcinoma from female never-smokers varies with histologic subtypes and age at diagnosis. Clin Cancer Res 18, 1947-1953 (2012).

10. Cha, M. J. et al. Micropapillary and solid subtypes of invasive lung adenocarcinoma: clinical predictors of histopathology and outcome. J Thorac Cardiovasc Surg 147, 921-928 e922 (2014).

11. Xu, L., Tavora, F. \& Burke, A. Histologic features associated with metastatic potential in invasive adenocarcinomas of the lung. Am J Surg Pathol 37, 1100-1108 (2013).

12. Russell, P. A. et al. Does lung adenocarcinoma subtype predict patient survival?: A clinicopathologic study based on the new International Association for the Study of Lung Cancer/American Thoracic Society/European Respiratory Society international multidisciplinary lung adenocarcinoma classification. J Thorac Oncol 6, 1496-1504 (2011).

13. Sakurai, H. et al. Differences in the prognosis of resected lung adenocarcinoma according to the histological subtype: a retrospective analysis of Japanese lung cancer registry data. Eur J Cardiothorac Surg 45, 100-107 (2014).

14. Yoshida, T. et al. Solid predominant histology predicts EGFR tyrosine kinase inhibitor response in patients with EGFR mutation-positive lung adenocarcinoma. J Cancer Res Clin Oncol 139, 1691-1700 (2013).

15. Woo, T. et al. Prognostic value of the IASLC/ATS/ERS classification of lung adenocarcinoma in stage I disease of Japanese cases. Pathol Int 62, 785-791 (2012)
16. de Bruin, E. C. et al. Reduced NF1 expression confers resistance to EGFR inhibition in lung cancer. Cancer Discov 4, 606-619 (2014).

17. Cancer Genome Atlas Research, N. Comprehensive molecular profiling of lung adenocarcinoma. Nature 511, 543-550 (2014)

18. Detterbeck, F. C., Boffa, D. J. \& Tanoue, L. T. The new lung cancer staging system. Chest 136, 260-271 (2009).

19. Pan, Y. et al. ALK, ROS1 and RET fusions in 1139 lung adenocarcinomas: A comprehensive study of common and fusion pattern-specific clinicopathologic, histologic and cytologic features. Lung Cancer 2, 121-126 (2014).

20. Wang, R. et al. RET fusions define a unique molecular and clinicopathologic subtype of non-small-cell lung cancer. J Clin Oncol 30, 4352-4359 (2012).

\section{Author contributions}

Y.Z., J.L. and R.W. designed this study, performed experiments and wrote the main manuscript. Y.L. and L.S. reviewed the pathologic slides and collected the pathologic data Y.J.P., D.C., H.C.H., H.L., T.Y., X.Y.L., Y.L.Z. and B.L. collected the clinical data, tumor samples and performed experiments. H.Q.C. and Y.H.S. designed and directed the overall project. All authors reviewed the manuscript.

\section{Additional information}

Competing financial interests: The authors declare no competing financial interests.

How to cite this article: Zhang, Y. et al. The prognostic and predictive value of solid subtype in invasive lung adenocarcinoma. Sci. Rep. 4, 7163; DOI:10.1038/srep07163 (2014).

This work is licensed under a Creative Commons Attribution-NonCommercialNoDerivs 4.0 International License. The images or other third party material in this article are included in the article's Creative Commons license, unless indicated otherwise in the credit line; if the material is not included under the Creative Commons license, users will need to obtain permission from the license holder in order to reproduce the material. To view a copy of this license, visit http:// creativecommons.org/licenses/by-nc-nd/4.0/ 